\title{
THE COMPUTATION OF THE NON-COMMUTATIVE GENERALIZATION OF THE A-POLYNOMIAL FOR THE FIGURE-EIGHT KNOT
}

\author{
RĂZVAN GELCA AND JEREMY SAIN
}

\begin{abstract}
The paper computes the noncommutative A-ideal of the figureeight knot, a noncommutative generalization of the A-polynomial. It is shown that if a knot has the same A-ideal as the figure-eight knot, then all colored Kauffman brackets are the same as those of the figure eight knot.
\end{abstract}

\section{INTRODUCTION}

The noncommutative A-ideal is a knot invariant that generalizes the A-polynomial of Cooper, Culler, Gillet, Long, and Shalen. It was introduced in the framework of quantum topology. Recall that the A-polynomial is computed from the pull back to the ring of polynomials, through a sequence of rational maps, of the ideal that determines the character variety of the knot complement. The noncommutative A-ideal is a deformation of this ideal with respect to a parameter, and is a finitely generated ideal in the quantum plane.

The purpose of this paper is to find the noncommutative A-ideal of the figureeight knot. The computation is based on skein operations in the knot complement and on the noncommutative trigonometry of the noncommutative torus. While the formulas we obtain are complicated and not at all illuminating, there are three observations that can be extracted from this work.

First, the fact that the noncommutative A-ideal of the figure-eight knot can be computed. Up to now only the A-ideals of the unknot, trefoil, and to some extent $(2,2 p+1)$-torus knots have been determined. But the complements of all these knots are Seifert fibered spaces, which is a true advantage when computing quantum invariants. The present paper is the first example of a computation of the noncommutative A-ideal for a hyperbolic knot.

Second, our methods can be adapted to give a pictorial way of computing the (classical) A-polynomial of the figure-eight knot. The work is done in the ring of functions on the $S L(2, \mathbb{C})$-character variety of the knot complement. It will be seen below that an important role is played by the characters of irreducible representations of $S L(2, \mathbb{C})$.

Finally, as a corollary of our work, we discovered that the noncommutative Aideal of the figure-eight knot determines all colored Kauffman brackets of this knot. More precisely, any knot that has the same A-ideal as the figure-eight knot has the same colored Kauffman brackets, in the zero framing of the knot. Recall that

Date: 1 December 2002.

1991 Mathematics Subject Classification. 57M27.

Key words and phrases. Kauffman bracket, A-polynomial, skein module. 
the colored Kauffman brackets are obtained by evaluating the classical Kauffman bracket on the colorings of the knot by Jones-Wenzl idempotents.

This property is a consequence of the so called "orthogonality relation" between the A-ideal and the Jones polynomial. It has been proved before that the noncommutative A-ideals of the unknot and the $(2,2 p+1)$-torus knots determine their colored Kauffman brackets. We now suggest the hypothesis that this property is true for all knots.

The paper is organized as follows. In Section 2 we recall the definitions of the A-polynomial and of the noncommutative A-ideal, together with some basic facts about the structure of the Kauffman bracket skein algebra of the torus. In Section 3 we explain briefly how the Kauffman bracket skein module of the complement of the figure-eight knot is computed, which is the content of an unpublished theorem of Bullock and Lofaro. In Section 4 we perform the skein computations that carry all specific information about the action of the Kauffman bracket skein algebra of the torus on the skein module of the knot complement. Section 5 computes the generators of the peripheral ideal of the knot, which is the left ideal sent to zero by the map from the skein algebra of the boundary torus to the skein module of the knot complement. This ideal determines the noncommutative A-ideal, exhibited in the next section. Section 7 contains what we consider the most important result of the paper, a theorem showing that the A-ideal of the figure-eight knot determines all its colored Kauffman brackets.

The authors would like to thank Fumikazu Nagasato for discovering and correcting an error that appeared in Proposition 5.4 below.

\section{Some facts about the A-Polynomial and the noncommutative A-IDEAL}

The computation of the A-polynomial of a knot is based on the following sequence of rational maps

$$
\mathbb{C} \times \mathbb{C} \hookleftarrow \mathbb{C}^{*} \times \mathbb{C}^{*} \rightarrow X\left(\mathbb{T}^{2}\right) \leftarrow X(M),
$$

where $X(M)$ and $X\left(\mathbb{T}^{2}\right)$ are the character varieties of $S L(2, \mathbb{C})$-representations of the fundamental group of the knot complement, respectively the torus. The first map is the inclusion, the second is the 2-1 covering map of the character variety, and the third is obtain by restricting representations to loops on the boundary of the knot complement. If we send $X(M)$ to $\mathbb{C} \times \mathbb{C}$ through this sequence, we obtain a closed algebraic set whose one dimensional part is determined by the A-polynomial (times $l-1)$ (see [5]).

Considering the rings of regular functions on these varieties, we obtain the dual sequence of maps

$$
\mathbb{C}[l, m] \hookrightarrow \mathbb{C}\left[l, l^{-1}, m, m^{-1}\right] \hookleftarrow \mathbb{C}\left[l, l^{-1}, m, m^{-1}\right]^{i n v} \rightarrow \mathcal{F}(X(M))
$$

where $\mathbb{C}\left[l, l^{-1}, m, m^{-1}\right]^{i n v}=\mathcal{F}\left(X\left(\mathbb{T}^{2}\right)\right)$ is the ring of functions on the character variety of the torus, which consists of the Laurent polynomials that are invariant under the simultaneous transformations $l \rightarrow l^{-1}$, and $m \rightarrow m^{-1}$; and $\mathcal{F}(X(M))$ is the ring of regular functions on the character variety of the knot complement. Let $I(K) \in \mathbb{C}\left[l, l^{-1}, m, m^{-1}\right]^{i n v}$ be the kernel of the third map of this sequence. Extending $I(K)$ to the ring of Laurent polynomials and then contracting it to $\mathbb{C}[l, m]$ yields a finitely generated ideal of polynomials. The radical of the one dimensional part of this ideal is generated by the A-polynomial (times $l-1$ ). 
Now recall that the Kauffman bracket skein module of a 3 -manifold $M$, denoted by $K_{t}(M)$, is the quotient of the free $\mathbb{C}\left[t, t^{-1}\right]$-module with basis the isotopy classes of links in $M$ by the submodule spanned by relations of the form $\left.\searrow-t \smile-t^{-1}\right)$ ( and $\bigcirc+t^{2}+t^{-2}$, where the links in each expression are identical except in a ball in which they look like depicted. Since in the computations below we will divide by polynomials, we slightly change the point of view, working over the field $\mathbb{C}(t)$ instead of the ring of Laurent polynomials $\mathbb{C}\left[t, t^{-1}\right]$. Note that in [7] $t$ was a complex number instead of a variable; all considerations below work in this setting, too, when $t$ is not a 48th root of unity. So our modules are in fact vector spaces, but for historical considerations we will keep the name.

At $t=-1, K_{-1}(M)$ endowed with the multiplication induced by the disjoint union of links, is a ring. As explained in [2] and [14, when reduced by $\sqrt{0}$, it is isomorphic to $\mathcal{F}(X(M))$, the ring of regular function on the $S L(2, \mathbb{C})$-character variety.

For the cylinder over a surface $\Sigma, K_{t}(\Sigma \times I)$ is an algebra. Here and below $I$ is the interval $[0,1]$. It was shown in [6] that the Kauffman bracket skein algebra of the cylinder over the torus is isomorphic to the algebra generated by noncommutative cosines in the noncommutative torus. The proof relies on the product-to-sum formula in $K_{t}\left(\mathbb{T}^{2} \times I\right)$, which will play an important role in this paper, so we remind it to the reader. Let $T_{n}(x)$ be the $n$th Chebyshev polynomial, $T_{0}(x)=2, T_{1}(x)=x$, $T_{n+1}(x)=x T_{n}(x)-T_{n-1}(x), n \in \mathbb{Z}$. For two integers $p$ and $q$ let $n$ be their greatest common divisor, $p^{\prime}=p / n, q^{\prime}=q / n$. The skein $(p, q)_{T}$ is by definition equal to $T_{n}\left(\left(p^{\prime}, q^{\prime}\right)\right)$ where the variable of the Chebyshev polynomial is the $p^{\prime} / q^{\prime}$ curve on the torus, and powers consist of parallel copies. Then $\left\{(p, q)_{T}, p, q \in \mathbb{Z}, p \geq 0\right\}$ is a basis of $K_{t}\left(\mathbb{T}^{2} \times I\right)$. The product-to-sum formula is

$$
(p, q)_{T} *(r, s)_{T}=t_{r s}^{\mid p q} \mid(p+r, q+s)_{T}+t^{-\left.\right|_{r s} ^{p q} \mid}(p-r, q-s)_{T} .
$$

The elements $t^{-p q} l^{p} m^{q}$ are the noncommutative exponentials of the noncommutative torus, so the map $(p, q)_{T} \rightarrow t^{-p q}\left(l^{p} m^{q}+l^{-p} m^{-q}\right)$ identifies $\frac{1}{2}(p, q)_{T}$ with a noncommutative cosine.

We can now deform the sequence of maps defining the A-polynomial to the following

$$
\mathbb{C}_{t}[l, m] \hookrightarrow \mathbb{C}_{t}\left[l, l^{-1}, m, m^{-1}\right] \hookleftarrow K_{t}\left(\mathbb{T}^{2} \times I\right) \rightarrow K_{t}(M)
$$

Here $\mathbb{C}_{t}[l, m]$ and $\mathbb{C}_{t}\left[l, l^{-1}, m, m^{-1}\right]$ are the rings of polynomials, respectively Laurent polynomials, in the noncommuting variables $l$ and $m$, satisfying $l m=t^{2} m l$. Let $I_{t}(K)$ be the kernel of the third map, which we call the peripheral ideal. It is a left ideal. If we extend it to Laurent polynomials, then contract it to $\mathbb{C}_{t}[l, m]$, we obtain a finitely generated left ideal of polynomials, called the noncommutative Aideal of the knot. It was introduced in [7] as a generalization of the A-polynomial. In this paper we compute it for the figure-eight knot.

At this point we would like to recall that in quantum mechanics $\mathbb{C}_{t}\left[l, l^{-1}, m, m^{-1}\right]$ is the algebra generated by the exponentials of the momentum and position operators and their inverses. This is the ring of trigonometric polynomials in the noncommutative torus. The ring $\mathbb{C}_{t}[l, m]$ is called the quantum plane.

For people interested ultimately in the A-polynomial we make the following remarks. The A-polynomial is usually determined by looking first at the representation variety of the fundamental group, then using elimination theory to obtain 
relations at the level of character variety. In this sense the computation is "geometric" - it is done at the level of the underlying geometric space.

Now if in the computations of this paper we substitute $t$ by -1 , then, with appropriate modifications to account for the problem with 48th roots of unity (see below), we obtain an "algebraic" computation of the A-polynomial. This means that we study the ring of functions on the character variety, instead of the character variety itself. A nice feature of this approach is that it can be done pictorially. A skein is identified with the function on the character variety defined by evaluating characters on that skein [4, 2], 14]. The sliding under a crossing corresponds to the element in the ideal of the variety that comes from the crossing, in the Wirtinger presentation of the knot. The Kauffman bracket skein relation at $t=-1$ corresponds to the Hamilton-Cayley identity for $S L(2, \mathbb{C})$. This method was applied in 12] for computing the A-polynomial of $(2,2 p+1)$-torus knots.

One should note that an important role in these computations is played by the polynomials $S_{n}(x)$, where $S_{0}(x)=1, S_{1}(x)=x, S_{n+1}(x)=x S_{n}(x)-S_{n-1}(x)$, $n \in \mathbb{Z}$. For $n<0, S_{n}(x)=-S_{-n-2}(x)$, while for $n>0, S_{n}(x)$ is the character of the $n+1$-dimensional irreducible representation of $S L(2, \mathbb{C})$. These polynomials satisfy $S_{n}-S_{n-2}=T_{n}$, where $T_{n}$ is the $n$th Chebyshev polynomial.

\section{The Kauffman Bracket Skein module of the Complement of the FIGURE-EIGHT KNOT}

It was shown in 3 that the Kauffman bracket skein module of the complement of the figure-eight knot is the $\mathbb{C}(t)$-module with basis $x^{n}, x^{n} y, x^{n} y^{2}, n \geq 0$, or equivalently $x^{n}, x^{n} z, x^{n} z^{2}, n \geq 0$, where $x, y$ and $z$ are depicted in Figure 3.1. Here and below, for two curves $\gamma_{1}$ and $\gamma_{2}$ when we write $\gamma_{1}^{n} \gamma_{2}^{m}$ we understand the disjoint union of $n$ parallel copies of the first curve and $m$ parallel copies of the second, all copies lying in (very thin) tubular neighborhoods of the initial curves.

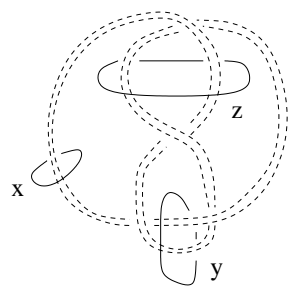

Figure 3.1.

Since [3] has not been published yet, we give here a brief overview of how the Kauffman bracket skein module of the complement of the figure-eight knot is computed. The complement of this knot is obtained by attaching a 2-handle to a genus two handlebody along the curve described in Figure 3.2. In the picture one should understand that the curve lies on the sphere, on viewer's side.

Represent the handlebody in the standard way, and push the curve inside slightly as in Figure 3.3. The two images represent the side view and the view from above of the handlebody.

The Kauffman bracket skein module of the handlebody, $K_{t}(H)$ is free with basis $x^{n} x^{\prime m} z^{k}, m, n, k \geq 0$, where $x, x^{\prime}, z$ are the dotted curves from Figure 3.3 [13. The 


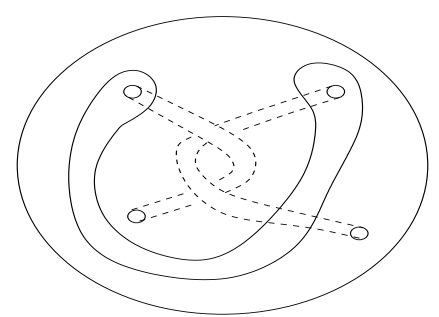

Figure 3.2.

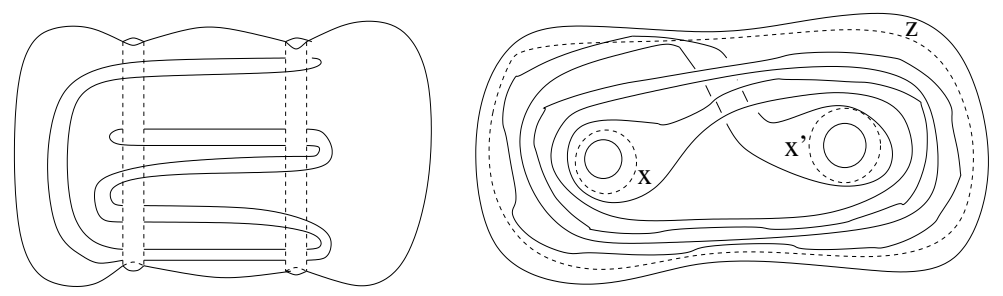

Figure 3.3.

skein module of the knot complement is obtained by factoring by the submodule corresponding to slidings of skeins through the 2-handle. This submodule is generated by slidings of basis elements. Clearly after attaching the handle $x$ and $x^{\prime}$ are isotopic. Thus we conclude that the Kauffman bracket skein module of the knot complement is obtained by factoring the free module with basis $x^{n} z^{m}$ by the submodule $J$ generated by $x^{n} z^{m}-x^{n} z^{m} \cdot s l(z)$, where $\operatorname{sl}(z)$ is the slide of $z$ through the 2-handle.

Now sliding through the 2-handle amounts to taking the banded sum with the attaching curve. Of course this can be done in infinitely many ways, but as explained in [1, we only need to consider one such banded sum, and we will always consider the one from Figure 3.4.
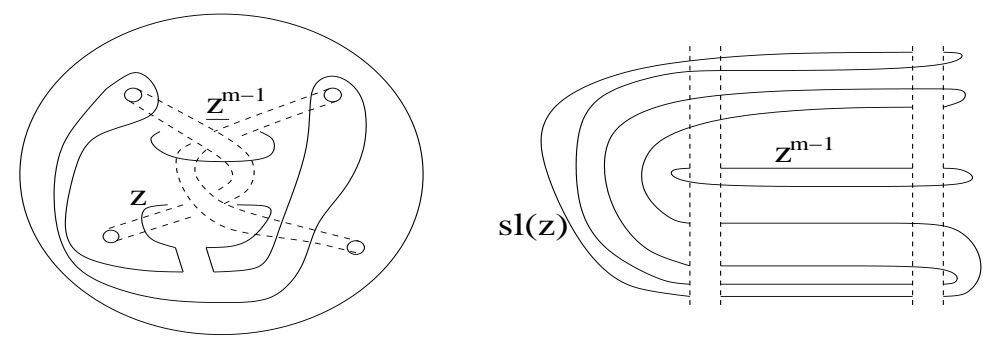

Figure 3.4.

It is not hard to see that up to a power of $t$, the element $x^{n} z^{m}-x^{n} z^{m-1} s l(z)$, $m \geq 1$ is a polynomial of $(m+3)$ rd degree in $z$ with the coefficient of $z^{m+3}$ equal to $x^{n}$ (just count how many times the curves wind "around" the handlebody and think what happens when you resolve the crossings). When we slide the trivial link (that is when we consider the attaching curve as a skein itself), we obtain a polynomial of the form $z^{4}+$ terms of lower degree. Through an unpleasant but routine computation one finds this polynomial, and also the ones for $z-\operatorname{sl}(z)$ and 
$z^{2}-z s l(z)$. Linear combinations of them yield polynomials of degrees 3,4 , and 5 in $z$. Hence $J$ is generated by polynomials of the form $x^{n} z^{m}+$ terms of lower degree in $z$, one for each $m \geq 3$. This implies that the Kauffman bracket skein module of the knot complement is free with basis $x^{n}, x^{n} z, x^{n} z^{2}, n \geq 0$.

Lemma 4.1 below shows that we can replace this basis by $x^{n}, x^{n} y, x^{n} z, n \geq 0$, where $x, y, z$ are the curves from Figure 3.1. This is the basis that we will work with.

We now make an important observation related to the amphichirality of the figure-eight knot. If we take the mirror image, the knot maps to itself. Since $x$ is a meridian, it stays unchanged, but $y$ maps to $z$ and $z$ to $y$. Also, under this symmetry $t$ changes to $t^{-1}$ and the skein $(p, q)_{T}$ on the boundary changes to $(p,-q)_{T}$.

\section{Skein computations in the Knot COMPlement}

Lemma 4.1. The following identities hold

$$
\begin{aligned}
& y^{2}=-t^{-2} x^{2} y-t^{-6} z-2 t^{-4} x^{2}+t^{-4}+1 \\
& z^{2}=-t^{2} x^{2} z-t^{6} y-2 t^{4} x^{2}+t^{4}+1
\end{aligned}
$$

Proof. To find $y^{2}$ start with the computation from Figure 4.1. This expression is

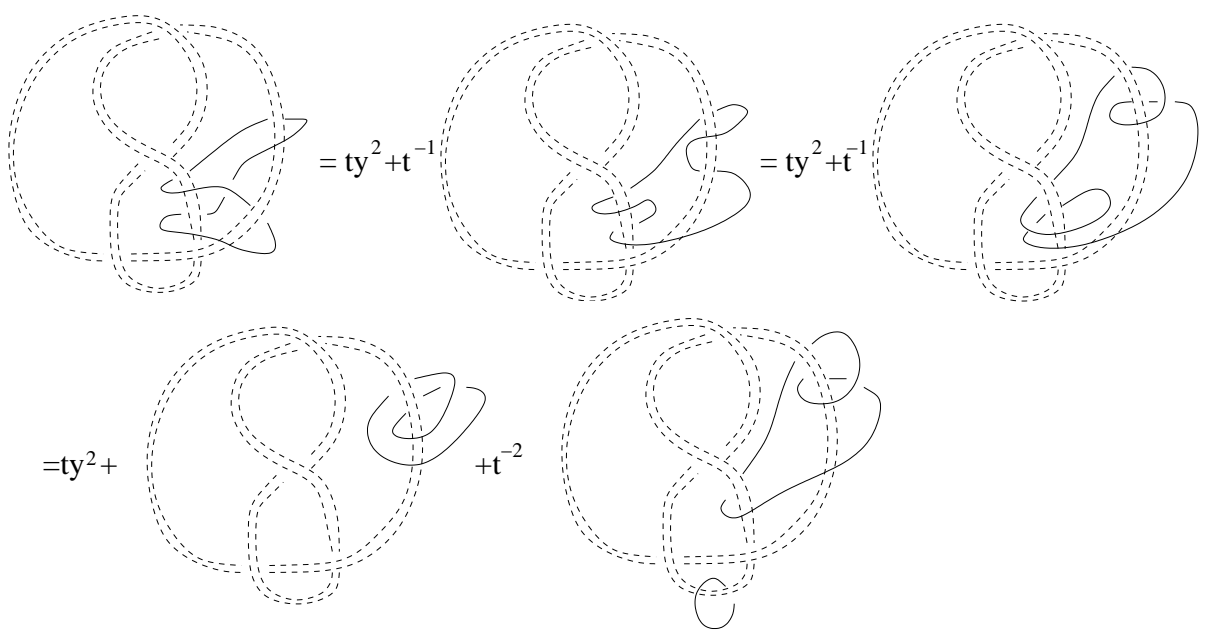

Figure 4.1.

equal to $t y^{2}+t x^{2}+t^{-1}\left(-t^{2}-t^{-2}\right)+t^{-1} x^{2} y+t^{-3} x^{2}$.

On the other hand, performing an isotopy on the strand we started with, we can then proceed as in Figure 4.2. Resolving the crossing we obtain $-t^{-3} x^{2}+t x^{2}-t^{-5} z$. The formula for $y^{2}$ now follows from the equality of these two expressions. Take the mirror image to obtain the formula for $z^{2}$.

Let $M$ be the complement of a regular neighborhood of the figure-eight knot complement. Below we will denote the image of $(p, q)_{T} \in K_{t}\left(\mathbb{T}^{2} \times I\right)$ through the morphism $K t\left(\mathbb{T}^{2} \times I\right) \rightarrow K_{t}(M)$ also by $(p, q)_{T}$. 


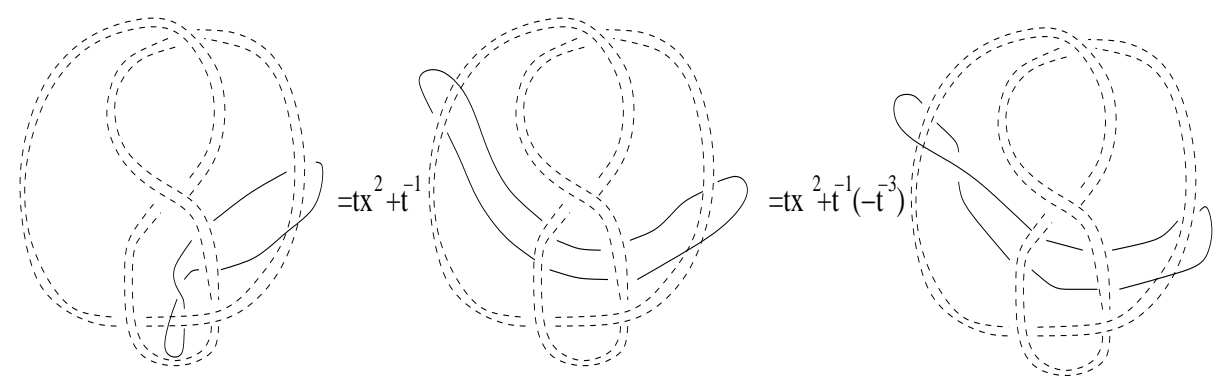

Figure 4.2.

Lemma 4.2. The images of $(1,0)_{T}$ and $(1,1)_{T}$ in the skein module of the figureeight knot complement are

$$
\begin{aligned}
& (1,0)_{T}=t^{4} x^{2} y+t^{-4} x^{2} z+\left(1-t^{4}\right) y+\left(1-t^{-4}\right) z+2\left(t^{2}+t^{-2}\right) x^{2}-t^{2}-t^{-2} \\
& (1,1)_{T}=t^{5} x^{3} y-2 t^{5} x y+t x z+t^{-3} x z+2 t^{3} x^{3}-3 t^{3} x+2 t^{-1} x
\end{aligned}
$$

Proof. We will only compute the image of $(1,0)_{T}$ in the skein module of the knot complement, the image of $(1,1)_{T}$ is then found in an analogous manner. The computation is described in Figure 4.3, where we start by resolving the two crossings on top.

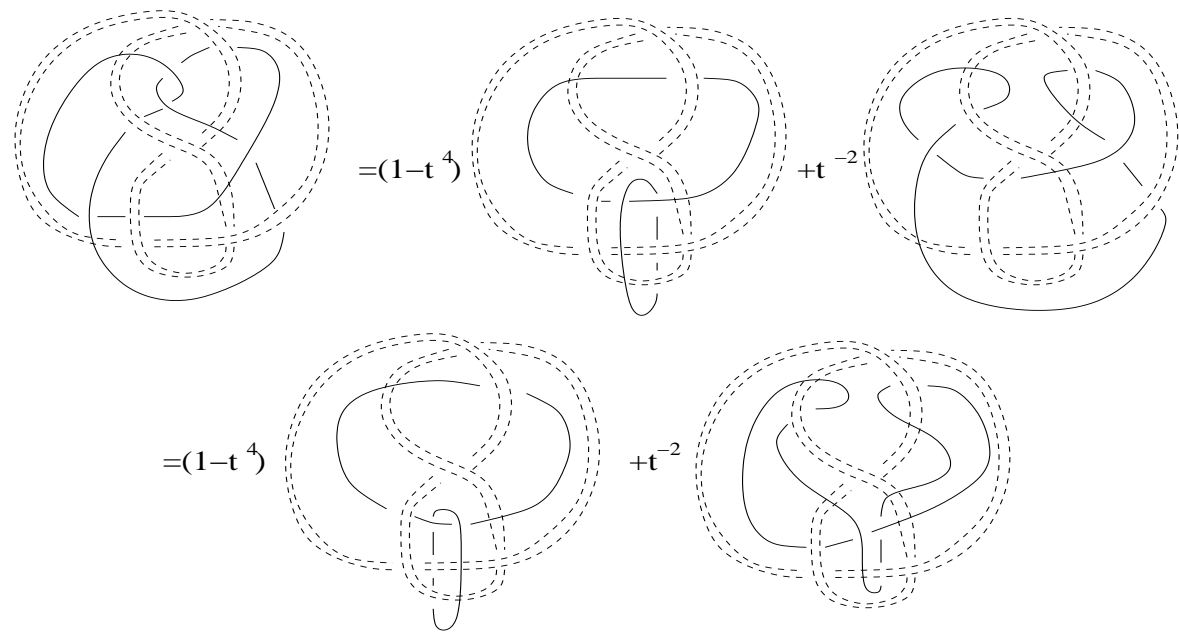

Figure 4.3.

Call the two terms on the second line A and B. Resolving the two crossings in $\mathrm{A}$ and performing the appropriate isotopies yields $-\left(1-t^{4}\right)\left(1-t^{-4}\right)\left(t^{2}-t^{-2}\right)+$ $t^{2}\left(1-t^{4}\right) y^{2}$. We point out the fact that the first term of this sum is obtained after replacing a trivial knot by $-t^{2}-t^{-2}$. With $\mathrm{B}$ the story is more complicated. We resolve the two crossings, then continue as in Figure 4.4. 

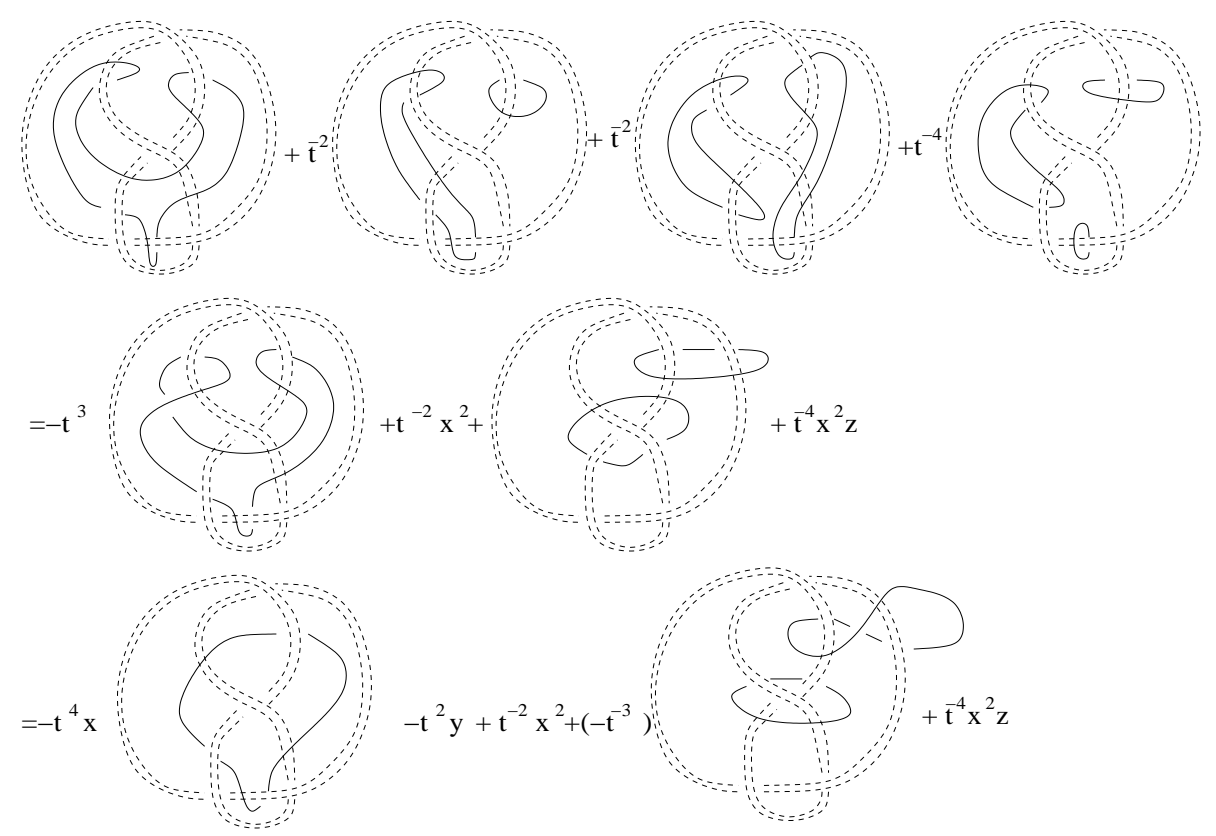

Figure 4.4.

In the end, after resolving the crossing, the term involving the second diagram is equal to $-t^{-4} x^{2} z-t^{-6} z^{2}$. In Figure 4.5 we compute the value of the remaining diagram (coefficient $-x$ excluded), which is equal to $-t^{-2} x y-t^{4} x$.

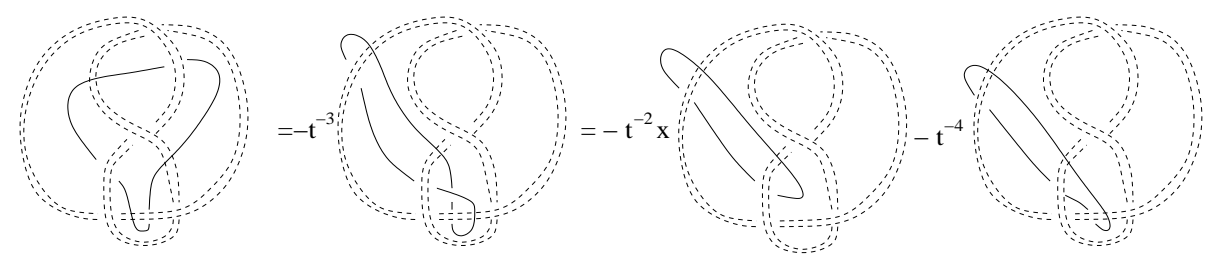

Figure 4.5.

It follows that $B=x^{2} y+t^{-4} x^{2} z+\left(1-t^{4}\right) y+4 t^{-2} x^{2}-t^{-2}-t^{2}$ After replacing $y^{2}$ and $z^{2}$ with their values given in Lemma 4.1 in $\mathrm{A}$ and adding $\mathrm{A}$ and $\mathrm{B}$ we obtain the desired formula.

To simplify the computations we denote $Y=t^{2} y+1, Z=t^{-2} z+1$. Also recall the polynomials $S_{n}(x)$, with $S_{0}(x)=1, S_{1}(x)=x, S_{n+1}(x)=x S_{n}(x)-S_{n-1}(x)$, $n \in \mathbb{Z}$.

Proposition 4.3. For any integer $q$, the following formula holds

$$
\begin{aligned}
t^{-q}(1, q)_{T}=\left(t^{2} S_{q+2}(x)-t^{-2} S_{q-2}(x)\right) Y & +\left(t^{2} S_{q}(x)-t^{-2} S_{q-4}(x)\right) Z \\
& +\left(t^{2} S_{q+2}(x)-t^{-2} S_{q-4}(x)\right) .
\end{aligned}
$$


Proof. For $q=0$ and $q=1$ this follows from Lemma 4.2 The product-to-sum formula yields the recursive relation

$$
(1, q+1)_{T}=t x(1, q)_{T}-t^{2}(1, q-1)_{T}
$$

and an inductive argument proves the formula in general.

Lemma 4.4. The action of the skein algebra of the boundary torus on the skein module of the figure-eight knot complement is determined by

$$
\begin{aligned}
& (1, q)_{T} Y=-t^{2}(1, q+2)_{T}+t^{q+2} T_{q+2}(x) Y+t^{q+2} T_{q}(x) \\
& (1, q)_{T} Z=-t^{-2}(1, q-2)_{T}+t^{q-2} T_{q-2}(x) Z+t^{q-2} T_{q}(x),
\end{aligned}
$$

where $q$ is any integer.

Proof. In the proof we prefer to work with $y$ instead of $Y=t^{2} y+1$. It suffices to prove the formula for the action on $y$, the action on $z$ is then determined by taking the mirror image. The idea is to compute "by hand" $(1,0)_{T} y$ and $(1,1)_{T} y$, and then use the product-to-sum formula to write the recurrence:

$$
(1, q+1)_{T} y=t x(1, q)_{T} y-t^{2}(1, q-1)_{T} y .
$$

We explain how $(1,0)_{T} y$ is determined and leave the case of $(1,1)_{T} y$ to the reader. We begin by resolving the crossing in $(1,0)_{T} y$ indicated by an arrow in Figure 4.6. After an isotopy, the second term becomes $-t^{-4} t^{6} y=-t^{2} y$. The first term is

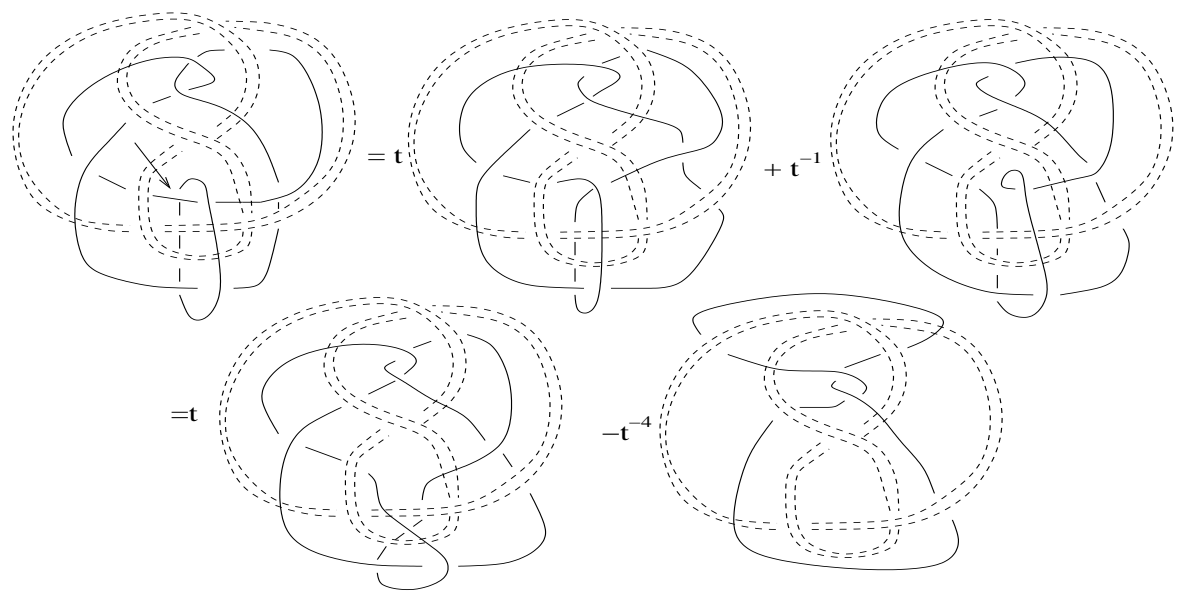

Figure 4.6.

nothing but $t^{2}\left(-t^{-3}(1,1)_{T} *(0,1)_{T}\right)+D$ where $D$ is the first diagram from Figure 4.7. The remaining part of Figure 4.7 computes the value of $D$. To conclude, the last two diagrams are computed by a method already presented in the proof of Lemma 4.2 (at the end of Figure 4.4). They are found to be $-t^{4} y\left(-t^{-2} x^{2}-t^{-4} z\right.$ ) and respectively $-t^{2}\left(-t^{-2} x^{2}-t^{-4} z\right)$. By adding all terms and using Lemma 4.1 and the product to-sum formula we obtain

$$
(1,0)_{T} y=-(1,2)_{T}-t^{-2}(1,0)_{T}+t^{2} T_{2}(x) y+x T_{1}(x)
$$

Finally, replacing $t^{2} y+1$ by $Y$ we obtain the desired result. 


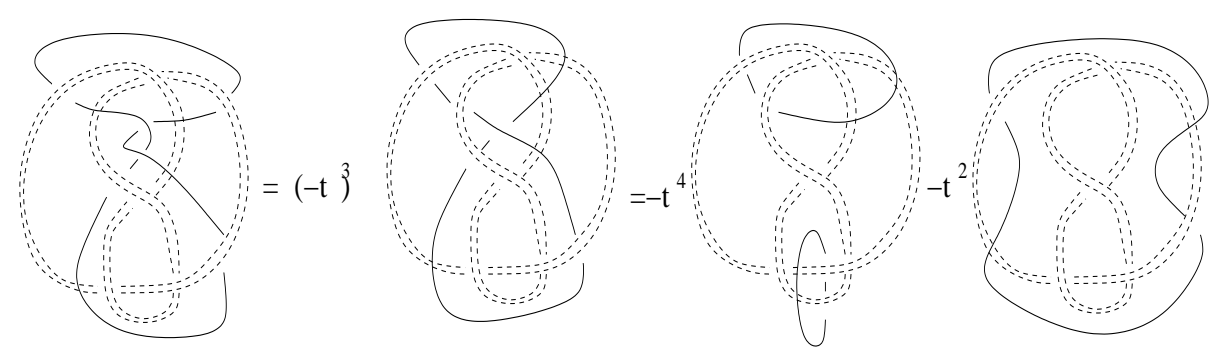

Figure 4.7.

Remark 4.5. All the information about the action of $K_{t}\left(\mathbb{T}^{2} \times I\right)$ on the skein module of the figure-eight knot complement is contained in Proposition 4.3 and Lemma 4.4 The action of some $(p, q)_{T} \in K_{t}\left(\mathbb{T}^{2} \times I\right)$ on a basis element of the form $x^{n} Y$ or $x^{n} Z$ can be computed using the product-to-sum formula.

\section{THE GENERATORS OF THE PERIPHERAL IDEAL}

We are now done with all necessary skein computations. From this moment on our work will be based on noncommutative trigonometry at the level of the Kauffman bracket skein algebra of the torus (hence in the noncommutative torus). Here is an immediate consequence of Lemma 4.2 that will be important in our computations.

Lemma 5.1. The following formulas are true

$$
\begin{aligned}
& {\left[S_{5}(x)-\left(1+t^{4}+t^{-4}\right) S_{1}(x)\right] Y=t^{-5}(1,3)_{T}-t^{3}(1,-1)_{T}+t^{4}(0,1)_{T}-(0,5)_{T}} \\
& {\left[S_{5}(x)-\left(1+t^{4}+t^{-4}\right) S_{1}(x)\right] Z=t^{5}(1,-3)_{T}-t^{-3}(1,1)_{T}+t^{-4}(0,1)_{T}-(0,5)_{T} .}
\end{aligned}
$$

We stress out that the lemma states that the left-hand sides are equal to the images in the skein module of the knot complement of the elements of the skein algebra of the torus written here on the right-hand side.

The next result, combined with Proposition 4.3 tells us what the image of $(2, q)_{T}$ is in the skein module of the figure-eight knot complement.

Lemma 5.2. For any integer $q$, the following identity holds

$$
\begin{aligned}
& t^{-2 q}(2, q)_{T}=\left[t^{8}(0, q+4)_{T}+\left(t^{4}+1\right)(0, q+2)_{T}+(0, q)_{T}\right] Y \\
& \quad+\left[t^{-8}(0, q-4)_{T}+\left(t^{-4}+1\right)(0, q-2)_{T}+(0, q)_{T}\right] Z \\
& \quad+t^{-q}\left[-t^{6}(1, q+4)_{T}-t^{-6}(1, q-4)_{T}\right] \\
& \quad+\left(t^{8}+1\right)(0, q+2)_{T}+\left(t^{4}+t^{-4}+1\right)(0, q)_{T}+\left(t^{-8}+1\right)(0, q-2)_{T} .
\end{aligned}
$$

Proof. By the product-to-sum formula

$$
t^{-2 q}(2, q)_{T}+(0, q)_{T}=t^{-q}(1, q)_{T} *(1,0)_{T} .
$$

Using Lemma 4.2 we see that this is further equal to

$$
\begin{aligned}
& t^{-q}(1, q)\left[\left(t^{2}(0,2)_{T}+\left(t^{2}+t^{-2}\right)\right) Y\right. \\
& \left.+\left(t^{-2}(0,2)_{T}+\left(t^{2}+t^{-2}\right)\right) Z+\left(t^{2}+t^{-2}\right)(0,2)_{T}+\left(t^{2}+t^{-2}\right)\right]
\end{aligned}
$$


or

$$
\begin{aligned}
& =t^{-q}\left[t^{4}(1, q+2)_{T} Y+(1, q-2)_{T} Y+\left(t^{2}+t^{-2}\right)(1, q)_{T} Y\right. \\
& +(1, q+2)_{T} Z+t^{-4}(1, q-2)_{T} Z+\left(t^{2}+t^{-2}\right)(1, q)_{T} Z \\
& \left.+\left(t^{4}+1\right)(1, q+2)_{T}+\left(1+t^{-4}\right)(1, q-2)_{T}+\left(t^{2}+t^{-2}\right)(1, q)_{T}\right] .
\end{aligned}
$$

Applying Lemma 4.4 we obtain the desired formula.

Proposition 5.3. For any integer $q$, one has

$$
\begin{aligned}
& t^{-2 q}(2, q)_{T}=\left[t^{-8} S_{q-4}(x)-t^{-4} S_{q}(x)+t^{-4} S_{q-2}(x)-t^{8} S_{q+2}(x)\right] Y \\
& \quad+\left[t^{-8} S_{q-4}(x)-t^{4} S_{q}(x)+t^{4} S_{q-2}(x)-t^{8} S_{q+2}(x)\right] Z \\
& \quad+t^{-q}\left[-t^{6}(1, q+4)_{T}-t^{-6}(1, q-4)_{T}+t^{4}(1, q+2)_{T}+t^{-4}(1, q-2)_{T}\right. \\
& \left.\quad+\left(t^{2}+t^{-2}\right)(1, q)_{T}\right]+\left[-t^{8} T_{q+4}(x)-t^{4} T_{q+2}(x)-t^{8} S_{q}(x)\right. \\
& \left.\quad+t^{-8} S_{q-2}(x)-t^{-4} T_{q-2}(x)-t^{-8} T_{q-4}(x)\right] .
\end{aligned}
$$

Proof. Recall that $(0, q)_{T}=T_{q}(x)$ and $T_{q}(x)=S_{q}(x)-S_{q-2}(x)$ for any integer $q$. The proposition then follows from Lemma 5.2 by applying the formulas from Proposition 4.3 to the terms that contain $(1, q \pm 2)_{T}$ and $(1, q)_{T}$.

Proposition 5.4. The elements

$$
\begin{aligned}
& t^{-6}(2,3)_{T}-t^{6}(2,-1)_{T}+t^{3}(1,7)_{T}-t(1,5)_{T} \\
& \quad+\left(-t^{11}+t^{3}-t^{-1}-t^{-5}\right)(1,3)_{T}+\left(t^{9}-t^{5}-t^{-7}\right)(1,1)_{T} \\
& \quad+\left(-t^{11}+2 t^{7}+t^{3}-t^{-1}+t^{-9}\right)(1,-1)_{T}+\left(t^{13}+t\right)(1,-3)_{T} \\
& \quad-t^{-1}(1,-5)_{T}+t^{8}(0,7)_{T}+\left(-2 t^{8}+t^{4}-t^{-4}\right)(0,5)_{T} \\
& \quad+\left(-t^{12}+t^{8}-t^{4}-1+t^{-4}\right)(0,3)_{T}+\left(t^{12}-t^{8}+1+t^{-4}\right)(0,1)_{T}
\end{aligned}
$$

and

$$
\begin{aligned}
& t^{6}(2,-3)_{T}-t^{-6}(2,1)_{T}+t^{-3}(1,-7)_{T}-t^{-1}(1,-5)_{T} \\
& \quad+\left(-t^{-11}+t^{-3}-t-t^{5}\right)(1,-3)_{T}+\left(t^{-9}-t^{-5}-t^{7}\right)(1,-1)_{T} \\
& \quad+\left(-t^{-11}+2 t^{-7}+t^{-3}-t+t^{9}\right)(1,1)_{T}+\left(t^{-13}+t^{-1}\right)(1,3)_{T} \\
& \quad-t(1,5)_{T}+t^{-8}(0,7)_{T}+\left(-2 t^{-8}+t^{-4}-t^{4}\right)(0,5)_{T} \\
& \quad+\left(-t^{-12}+t^{-8}-t^{-4}-1+t^{4}\right)(0,3)_{T}+\left(t^{-12}-t^{-8}+1+t^{4}\right)(0,1)_{T}
\end{aligned}
$$

are in the peripheral ideal of the figure-eight knot.

Proof. Using Proposition 5.3 we obtain

$$
\begin{aligned}
& t^{-6}(2,3)_{T}=\left[t^{-8} S_{-1}(x)-t^{-4} S_{3}(x)+t^{-4} S_{1}(x)-t^{8} S_{5}(x)\right] Y \\
& +\left[t^{-8} S_{-1}(x)-t^{4} S_{3}(x)+t^{4} S_{1}(x)-t^{8} S_{5}(x)\right] Z \\
& +t^{-3}\left[-t^{6}(1,7)_{T}-t^{-6}(1,-1)_{T}+t^{4}(1,5)_{T}+t^{-4}(1,1)_{T}+\left(t^{2}+t^{-2}\right)(1,3)_{T}\right] \\
& +\left[-t^{8} T_{7}(x)-t^{4} T_{5}(x)-t^{8} S_{3}(x)+t^{-8} S_{1}(x)-t^{-4} T_{1}(x)-t^{-8} T_{-1}(x)\right] .
\end{aligned}
$$


Using Lemma 5.1 and the fact that $S_{-n}=-S_{n-2}, S_{-1}=0$, and $T_{-n}=T_{n}$, we obtain

$$
\begin{aligned}
& t^{-6}(2,3)_{T}=\left[-t^{-4} S_{3}(x)+\left(t^{-4}-t^{12}-t^{4}-t^{8}\right) S_{1}(x)\right] Y \\
& \quad+\left[-t^{-4} S_{3}(x)+\left(-t^{12}-t^{8}\right) S_{1}(x)\right] Z \\
& \quad+\left[-t^{3}(1,7)_{T}+t(1,5)_{T}+\left(t^{-1}+t^{-5}-t^{3}\right)(1,3)_{T}+\left(t^{-7}+t^{5}\right)(1,1)_{T}\right. \\
& \left.\quad+\left(t^{11}-t^{-9}\right)(1,-1)_{T}-t^{13}(1,-3)_{T}\right]+\left[-t^{8}(0,7)_{T}\right. \\
& \left.\quad+\left(2 t^{8}-t^{4}\right)(0,5)_{T}-t^{8}(0,3)_{T}-\left(t^{8}+t^{4}+t^{-4}+t^{12}\right)(0,1)_{T}\right] .
\end{aligned}
$$

A similar computation yields

$$
\begin{aligned}
& t^{6}(2,-1)_{T}=\left[-t^{-4} S_{3}(x)-\left(t^{12}+1\right) S_{1}(x)\right] Y \\
& \quad+\left[-t^{-4} S_{3}(x)-\left(t^{12}+t^{8}\right) S_{1}(x)\right] Z \\
& \quad+\left[-t^{11}(1,3)_{T}-t^{-1}(1,-5)_{T}+t^{11}(1,1)_{T}+t(1,-3)_{T}+\left(t^{7}+t^{3}\right)(1,-1)_{T}\right] \\
& \quad+\left[\left(-t^{12}-1\right)(0,3)_{T}-t^{-4}(0,5)_{T}+\left(t^{-4}-t^{8}\right)(0,1)_{T}\right] .
\end{aligned}
$$

Now when we subtract the two, everything is in the image of the $K_{t}\left(\mathbb{T}^{2} \times I\right)$ except for $\left(t^{-4}-t^{4}-t^{8}+1\right) S_{1}(x) Y+\left(t^{-4}-t^{4}\right) S_{3}(x) Z$. We can rewrite this as

$$
\left(-t^{6}-t^{-2}\right)\left[\left(t^{2} S_{1}(x)-t^{-2} S_{-3}(x)\right) Y-\left(t^{2} S_{-1}(x)-t^{-2} S_{-5}(x)\right) Z\right]
$$

and then we recognize the $Y, Z$-parts in the formula for $(1,-1)_{T}$ as given by Proposition 4.2 Using that formula and moving everything to the right we obtain an element in $K_{t}\left(\mathbb{T}^{2} \times I\right)$ that is mapped to zero in the skein module of the knot complement. This is exactly the first element from the statement.

The second element is the mirror image of the first, and since the figure eight knot is amphichiral, it also belongs to the peripheral ideal.

For a nonnegative integer $n$ let us denote by $V(n)$ the intersection of the peripheral ideal $I_{t}(K)$ with the $\mathbb{C}(t)$-submodule of $K_{t}\left(\mathbb{T}^{2} \times I\right)$ that is generated by elements of the form $(p, q)_{T}, 0 \leq p \leq n$. We point out that $V(n)$ is not an ideal.

Lemma 5.5. The elements $x Y$ and $x Z$ are in the image of $V(2)$ in the the skein module of the figure-eight knot complement.

Proof. If in the formula for $(2,-1)_{T}$ obtained in the proof of Proposition 5.4 we add on both sides $t^{-3}(1,-1)_{T}+t^{-7}(1,1)_{T}$ and expand these two terms on the right-hand side using Lemma 4.3 we obtain

$$
\begin{aligned}
& t^{6}(2,-1)_{T}+t^{-3}(1,-1)_{T}+t^{-7}(1,1)_{T}=-t^{-4}\left(t^{16}-1\right) S_{1}(x) Y \\
& -t^{-4}\left(t^{16}-1\right)\left(t^{-4}-1\right) S_{1}(x) Z-t^{-1}(1,-5)_{T}+t^{-1}(1,-3)_{T}+\left(t^{7}+t^{3}\right)(1,-1)_{T} \\
& +t^{9}(1,1)_{T}-t^{11}(1,3)_{T}-t^{-4}(0,5)_{T}+\left(t^{-4}-t^{12}-1\right)(0,3)_{T} \\
& +\left(-t^{8}-1-t^{-4}-t^{-8}\right)(0,1)_{T} .
\end{aligned}
$$

Similarly

$$
\begin{aligned}
& t^{-6}(2,1)_{T}+t^{3}(1,1)_{T}+t^{7}(1,-1)_{T}=t^{-12}\left(t^{16}-1\right) S_{1}(x) Z \\
& t^{-12}\left(t^{16}-1\right)\left(t^{4}-1\right) S_{1}(x) Y-t(1,5)_{T}+t(1,3)_{T} \\
& +\left(t^{-7}+t^{-3}\right)(1,1)_{T}+t^{-9}(1,-1)_{T}-t^{-11}(1,-3)_{T} \\
& -t^{4}(0,5)_{T}+\left(t^{4}-t^{-12}-1\right)(0,3)_{T}+\left(-t^{-8}-1-t^{4}-t^{8}\right)(0,1)_{T} .
\end{aligned}
$$


Recall that $S_{1}(x)=x$. Multiplying the second equation by $t^{4}-t^{8}$, adding the two, and dividing by $\left(t^{16}-1\right)\left(t^{-8}-t^{-4}+1\right)$ we obtain

$$
\begin{aligned}
x Y & =\frac{1}{\left(t^{16}-1\right)\left(1-t^{-4}+t^{-8}\right)}\left[\left(t^{-2}-t^{2}\right)(2,1)_{T}+t^{6}(2,-1)_{T}+\left(t^{5}-t^{9}\right)(1,5)_{T}\right. \\
& +\left(t^{11}+t^{9}-t^{5}\right)(1,3)_{T}+\left(-t^{11}-t^{9}+t^{7}-t^{5}-t^{-3}+t^{-7}\right)(1,1)_{T} \\
& +\left(t^{-3}-t^{15}+t^{11}-t^{7}-t^{3}+t^{-1}-t^{-5}\right)(1,-1)_{T} \\
& +\left(-t^{-1}-t^{-3}+t^{-7}\right)(1,-3)_{T}+t^{-1}(1,-5)_{T}+\left(-t^{12}+t^{8}+t^{-4}\right)(0,5)_{T} \\
& \left.+\left(2 t^{12}-2 t^{8}+t^{4}-2 t^{-4}+t^{-8}+1\right)(0,3)_{T}+\left(-t^{16}+t^{8}+t^{4}+2 t^{-4}+t^{-8}\right)(0,1)_{T}\right] .
\end{aligned}
$$

Taking the mirror image we obtain

$$
\begin{aligned}
& x Z=\frac{1}{\left(t^{-16}-1\right)\left(1-t^{4}+t^{8}\right)}\left[\left(t^{2}-t^{-2}\right)(2,-1)_{T}+t^{-6}(2,1)_{T}+\left(t^{-5}-t^{-9}\right)(1,-5)_{T}\right. \\
& \quad+\left(t^{-11}+t^{-9}-t^{-5}\right)(1,-3)_{T}+\left(-t^{-11}-t^{-9}+t^{-7}+t^{-5}-t^{3}+t^{7}\right)(1,-1)_{T} \\
& \quad+\left(t^{3}-t^{-15}+t^{-11}-t^{-7}-t^{-3}+t-t^{5}\right)(1,1)_{T} \\
& \quad+\left(-t-t^{3}+t^{7}\right)(1,3)_{T}+t(1,5)_{T}+\left(t^{-12}+t^{-8}+t^{4}\right)(0,5)_{T} \\
& \left.\quad+\left(2 t^{-12}-2 t^{-8}+t^{-4}-2 t^{4}+t^{8}+1\right)(0,3)_{T}+\left(-t^{-16}+t^{-8}+t^{-4}+2 t^{4}+t^{8}\right)(0,1)_{T}\right] .
\end{aligned}
$$

From now on $x Y$ and $x Z$ will be identified with the above preimages in the skein algebra of the boundary torus.

Proposition 5.6. The element

$$
\begin{aligned}
& -t(3,1)_{T}+\left(t^{16}-1\right)\left(t^{4}+1\right) t^{-2}(0,2)_{T}(x Y)+\left(t^{16}-1\right)\left(t^{4}+1\right)\left(t^{-6}-t^{-2}\right)(x Y) \\
& +\left(t^{16}-1\right) t^{-2}(0,2)_{T}(x Z)+\left(t^{16}-1\right)\left(t^{-6}-t^{-10}\right)(x Z) \\
& -t^{12}(2,5)_{T}+t^{8}(2,3)_{T}+\left(1+t^{4}-t^{10}\right)(2,1)_{T}+\left(t^{-4}-t^{12}\right)(2,-1)_{T}-t^{-8}(2,-3)_{T} \\
& -t^{15}(1,5)_{T}+\left(t^{-3}-t^{9}-t^{17}\right)(1,3)_{T}+\left(t^{-5}-t^{7}-2 t^{11}-2 t^{15}+t^{-1}\right)(1,1)_{T} \\
& \left(t^{-7}-t^{-3}-t^{-1}-t^{5}-t^{13}\right)(1,-1)_{T}+\left(t^{-9}-t^{-5}-t^{3}\right)(1,-3)_{T}-t^{5}(1,-5)_{T} \\
& -\left(t^{2}-t^{-2}\right)(0,3)_{T}+\left(t^{18}+t^{14}+2 t^{10}-t^{8}+t^{6}-2 t^{-6}-t^{-10}\right)(0,1)_{T}
\end{aligned}
$$

is in the peripheral ideal of the figure-eight knot.

Note that $x Y$ and $x Z$ were shown to be in the image of the skein algebra of the boundary, and it follows that $(0,2)_{T}(x Y)$ and $(0,2)_{T}(x Z)$ are in the image of this algebra, too.

Proof. By the product-to-sum formula we have

$$
t(3,1)_{T}-(1,0)_{T} *(2,1)_{T}+t^{-1}(1,1)_{T}=0 .
$$

Substitute in this $(1,0)_{T}$ using Lemma 4.2 and $(2,1)_{T}$ using the formula deduced in the proof of Proposition 5.5

Lemma 5.7. The following equalities hold

$$
\begin{aligned}
& (0,2)_{T} Y=(0,1)_{T}(x Y)-2 Y \\
& (0,2)_{T} Z=(0,1)_{T}(x Z)-2 Z .
\end{aligned}
$$


Proof. Since $x Y=(0,1)_{T} Y$ and $(0,0)_{T}=2$ the conclusion follows from

$$
(0,2)_{T} Y=(0,1)_{T} *(0,1)_{T} Y-(0,0)_{T}
$$

and its mirror image.

Lemma 5.8. One has the following formulas

$$
\begin{aligned}
Y & =\frac{1}{2\left(t^{-8}-t^{4}\right)\left(t^{4}-1\right)}\left[\left(t^{4}+t^{-4}+1-2 t^{8}-t^{-8}\right)(0,1)_{T}(x Y)\right. \\
& +\left(t^{-4}+1-t^{4}-t^{8}\right)(0,1)_{T}(x Z)-(2,0)_{T}-t^{6}(1,4)_{T}-t^{-6}(1,-4)_{T} \\
& +t^{4}(1,2)_{T}+t^{-4}(1,-2)_{T}+\left(t^{6}-t^{-6}\right)(1,0)_{T}-\left(t^{8}+t^{-8}\right)(0,4)_{T} \\
& \left.+\left(t^{-4}-t^{4}+2-t^{8}+t^{-8}\right)(0,2)_{T}+\left(2 t^{-4}+2-2 t^{8}\right)\right]
\end{aligned}
$$

and

$$
\begin{aligned}
Z & =\frac{1}{2\left(t^{8}-t^{-4}\right)\left(t^{-4}-1\right)}\left[\left(t^{-4}+t^{4}+1-2 t^{-8}-t^{8}\right)(0,1)_{T}(x Z)\right. \\
& +\left(t^{4}+1-t^{-4}-t^{-8}\right)(0,1)_{T}(x Y)-(2,0)_{T}-t^{6}(1,4)_{T}-t^{-6}(1,-4)_{T} \\
& +t^{4}(1,2)_{T}+t^{-4}(1,-2)_{T}+\left(t^{-6}-t^{6}\right)(1,0)_{T}-\left(t^{8}+t^{-8}\right)(0,4)_{T} \\
& \left.+\left(t^{4}-t^{-4}+2-t^{-8}+t^{8}\right)(0,2)_{T}+\left(2 t^{4}+2-2 t^{-8}\right)\right]
\end{aligned}
$$

Proof. Using the product-to-sum formula we obtain

$$
\begin{aligned}
& (2,0)_{T}=-\left(t^{8}+t^{-8}\right)(0,2)_{T} Y-\left(2 t^{-4}+t^{8}+t^{-8}\right) Y-\left(t^{8}+t^{8}\right)(0,2)_{T} Z \\
& -\left(2 t^{4}+t^{8}+t^{-8}\right) Z-t^{6}(1,4)_{T}-t^{-6}(1,-4)_{T}+t^{4}(1,2)_{T}+t^{-4}(1,-2)_{T} \\
& +\left(t^{2}+t^{-2}\right)(1,0)_{T}-t^{8}(0,4)_{T}-t^{4}(0,2)_{T}-t^{8}-t^{-8}-t^{-4}(0,2)_{T}-t^{-8}(0,4)_{T} \\
& \quad \quad=-\left(t^{6}+t^{2}+t^{-2}-t^{-6}\right)\left(t^{-2}-t^{2}\right) Y-\left(t^{-6}+t^{-2}+t^{2}-t^{6}\right)\left(t^{2}+t^{-2}\right) Z \\
& -\left(t^{8}+t^{-8}\right)(0,1)_{T}(x Y)-\left(t^{8}+t^{-8}\right)(0,1)_{T}(x Z) \\
& -t^{6}(1,4)_{T}-t^{-6}(1,-4)_{T}+t^{4}(1,2)_{T}+t^{-4}(1,-2)_{T}+\left(t^{2}+t^{-2}\right)(1,0)_{T} \\
& -\left(t^{8}+t^{-8}\right)(0,4)_{T}-\left(t^{4}+t^{-4}\right)(0,2)_{T}-\left(t^{8}+t^{-8}\right) .
\end{aligned}
$$

If we combine this with

$$
\begin{aligned}
(1,0)_{T}= & t^{2}(0,1)_{T}(x Y)+t^{-2}(0,1)_{T}(x Z)+\left(t^{-2}-t^{2}\right) Y+\left(t^{2}-t^{-2}\right) Z \\
& +\left(t^{2}+t^{-2}\right)(0,2)_{T}+\left(t^{2}+t^{-2}\right),
\end{aligned}
$$

we obtain a system of two equations in the unknowns $Y$ and $Z$. Solving it we obtain the formulas from the statement.

Corollary 5.9. The Kauffman bracket skein module of the complement of the figureeight knot is peripheral. Moreover, it is the image of $V(2)$.

Proof. $Y$ and $Z$ are in $V(2)$ by Lemma 5.8 . Since $V(2)$ is closed under multiplication on the left by $(0,1)_{T}$ it follows that $x^{n}, x^{n} Y$ and $x^{n} Z$ are in the image of $V(2)$ for all nonnegative integers $n$. But by [3] these elements form a basis of the skein module of the knot complement. 
Proposition 5.10. The element

$$
\begin{aligned}
& -t^{3}(3,0)_{T}-t^{-7}(1,4)_{T} Y-t^{-13}(1,-2)_{T} Y-\left(t^{7}+t^{-9}+t^{-5}\right)(1,2)_{T} Y \\
& -\left(t^{5}+t^{-11}+t^{-7}\right)(1,0)_{T} Y-t^{-7}(1,4)_{T} Z-t^{-13}(1,-2)_{T} Z \\
& -\left(t^{7}+t^{-9}+t^{3}\right)(1,2)_{T} Z-\left(t^{5}+t^{-11}+t\right)(1,0)_{T} Z \\
& -t^{7}(2,4)_{T}-t^{-13}(2,-4)_{T}+t^{3}(2,2)_{T}+t^{9}(2,-2)_{T}+\left(t^{-1}+t^{-5}\right)(2,0)_{T} \\
& -\left(t^{9}-t^{-3}\right)(1,4)_{T}-\left(t^{3}+t^{-3}+t^{-9}\right)(1,-2)_{T}-t^{-5}(1,6)_{T}-t^{-15}(1,-4)_{T} \\
& +\left(t^{-9}-t^{3}\right)(1,2)_{T}+\left(t^{-11}-t\right)(1,0)_{T}-t^{-1}(0,6)_{T}+t^{-1}(0,4)_{T}+t^{-1}(0,2)_{T}+2 t^{3} .
\end{aligned}
$$

is in the peripheral ideal of the figure-eight knot.

Proof. The proof is analogous to the one given to Proposition [5.6] in this case one starts with

$$
(3,0)_{T}-(1,0)_{T} *(2,0)_{T}+(1,0)_{T}=0 .
$$

Theorem 5.11. The four elements described in Propositions 5.4, 5.6, and 5.10 generate the peripheral ideal of the figure-eight knot.

Proof. (1) First let us show that there are no nontrivial elements in $V(1) \cap I_{t}(K)$. Assume the contrary, namely that there exist finitely many $c_{q}$, not all equal to zero, such that $\sum_{q} c_{q} t^{-q}(1, q)_{T}$ is in the peripheral ideal. The fact that $S_{n}(x), S_{n}(x) Y$, $S_{n}(x) Z, n \geq 0$ form a basis of the skein module of the knot complement, combined with Lemma 4.3 implies that

$$
\begin{gathered}
\sum_{q} c_{q}\left(t^{2} S_{q+2}(x)-t^{-2} S_{q-2}(x)\right)=0 \\
\sum_{q} c_{q}\left(t^{2} S_{q}(x)-t^{-2} S_{q-4}(x)\right)=0 \\
\sum_{q} c_{q}\left(t^{2} S_{q+2}(x)-t^{-2} S_{q-4}(x)\right)=0 .
\end{gathered}
$$

as the first is the coefficient of $Y$, the second is the coefficient of $Z$ and the third is the free term in the formula of $\sum_{q} c_{q} t^{-q}(1, q)_{T}$. Subtracting the first from the last and the second from the last we obtain

$$
t^{-2} \sum_{q} c_{q} T_{q-2}(x)=0 \text { and } t^{2} \sum_{q} c_{q} T_{q+2}(x)=0 .
$$

Since the Chebyshev polynomials $T_{n}, n \geq 0$ form a basis of the vector space of polynomials, it follows from the first relation that $c_{q+2}=c_{-q+2}$, and from the second that $c_{q-2}=c_{-q-2}$, for all $q$. This is impossible unless all $c_{q}$ are equal to zero, a contradiction. Hence the claim is true.

(2) Now let us show that

$$
V(2) \cap I_{t}(K)=\left\{\alpha g_{1}+\beta g_{2}, \alpha, \beta \in \mathbb{C}(t)[x]\right\}
$$

where $g_{1}$ and $g_{2}$ are the two elements exhibited in the statement of Proposition 5.4.

Reduce the $\mathbb{C}(t)[x]$-modules modulo $V(1)$. Then

$$
t^{-6}(2,3)_{T}-t^{6}(2,-1)_{T} \equiv 0 \quad \text { and } \quad t^{6}(2,-3)_{T}-t^{-6}(2,1)_{T} \equiv 0 .
$$


Multiplying on the left by $(0,1)_{T}$ and using the product-to-sum formula we can prove inductively that for all integers $p$,

$$
(2,2 p+1)_{T}-t^{12}(2,2 p-3)_{T} \equiv 0 \quad(\bmod V(1))
$$

and

$$
(2,2 p+2)_{T}+t^{4}(2,2 p)_{T}-t^{12}(2,2 p-2)_{T}-t^{6}(2,2 p-4)_{T} \equiv 0 \quad(\bmod V(1)) .
$$

Assume that there exists an element in $V(2) \cap I_{t}(K)$ not generated by $g_{1}$ and $g_{2}$. Subtracting terms generated by $g_{1}$ and $g_{2}$ of the form $(2,2 p+1)_{T}-t^{12}(2,2 p-$ $3)_{T}+v_{2 p+1}$ or $(2,2 p+2)_{T}+t^{4}(2,2 p)_{T}-t^{12}(2,2 p-2)_{T}-t^{6}(2,2 p-4)_{T}+v_{2 p+2}$ with $v_{2 p+1}, v_{2 p+2} \in V(1)$, we can reduce such an element to one that is a linear combination of $(2,2)_{T},(2,1)_{T},(2,0)_{T},(2,-1)_{T},(2,-2)_{T}$. Examining the formulas in Propositions 4.3 and 5.3 we see that parity implies that the "even" part and the "odd" part can be separated. This means that there must be an element in $V(2) \cap I_{t}(K)(\bmod V(1))$ of the form $a(2,1)_{T}+b(2,-1)_{T}$, and one of the form $c(2,2)_{T}+d(2,0)_{T}+e(2,-2)_{T}$. We will show that $a=b=c=d=e=0$.

By Proposition 5.3 the fact that $a(2,1)_{T}+b(2,-1)_{T}$ is in the peripheral ideal modulo $V(1)$ means that

$$
\begin{aligned}
& {\left[-\left(t^{-4} a+t^{4} b\right) S_{3}(x)-\left(t^{12} a+a+t^{-12} b+t^{-8} b\right) S_{1}(x)\right] Y} \\
& \quad+\left[-\left(t^{-4} a+t^{4} b\right) S_{3}(x)-\left(t^{12} a+t^{8} a-t^{-12} b+b\right) S_{1}(x)\right] Z
\end{aligned}
$$

is in $V(1)$. Let it be equal to $\sum_{q} c_{q} t^{-q}(1, q)_{T}$. By parity we see that we can assume $c_{q}=0$ for $q$ even. Since

$$
\begin{aligned}
& \sum_{q} c_{q} t^{-q}(1, q)_{T}=\left(t^{2} c_{q} S_{q+2}(x)-t^{-2} c_{q} S_{q-2}(x)\right) Y \\
& +\left(t^{2} c_{q} S_{q}(x)-t^{-2} c_{q} S_{q-4}(x)\right) Z+c_{q}\left(t^{2} S_{q+2}(x)-t^{-2} S_{q-4}(x)\right) .
\end{aligned}
$$

$q$ can only be equal to $3,1,-1,-3$. Indeed, if we denote by $q_{0}$ and $q_{1}$ the largest and the smallest of the $q$ 's, then for $S_{q_{0}+2}(x) Y$ and $S_{q_{1}-2}(x) Y$ to cancel out one should have $q_{0}+2=-\left(q_{1}-2\right)-2$, and for $S_{q_{0}}(x) Z$ and $S_{q_{1}-4}(x) Z$ to cancel out one should have $q_{0}=-\left(q_{1}-4\right)-2$. So on the one hand $q_{0}=-q_{1}-2$ and on the other $q_{0}=-q_{1}+2$, and these two cannot hold simultaneously.

Let us now look at

$$
c_{3} t^{-3}(1,3)_{T}+c_{1} t^{-1}(1,1)_{T}+c_{-1} t(1,-1)_{T}+c_{-3} t^{3}(1,-3)_{T} .
$$

For the coefficients of $Y$ and $Z$ to be polynomials of third degree in $x$ one should have $c_{3}=c_{-3}=0$. So we are left with $c_{1} t^{-1}(1,1)_{T}+c_{-1} t(1,-1)_{T}$. Equating the coefficients of the basis elements $S_{3}(x) Y, S_{1}(x) Y, S_{3}(x) Z$ and $S_{1}(x) Z$ we obtain

$$
\begin{aligned}
& c_{1} t^{2}=-\left(t^{-4} a+t^{4} b\right) \\
& \left(-c_{1} t^{-2}+c_{-1} t^{2}+c_{-1} t^{-2}\right)=-\left(t^{12} a+a+t^{-12} b+t^{-8} b\right) \\
& c_{-1} t^{-2}=-\left(t^{-4} a+t^{4} b\right) \\
& \left(t^{2} c_{-1}+t^{-2} c_{-1}\right)=-\left(t^{12} a+t^{8} a-t^{-12} b+b\right) .
\end{aligned}
$$

This is a linear homogeneous system in the unknowns $a, b, c_{1}, c_{-1}$. An easy computer check shows that the determinant is different from zero, so the system has the unique solution $a=b=c_{1}=c_{-1}=0$.

The argument for $c(2,2)_{T}+d(2,0)_{T}+e(2,-2)_{T}$ is similar, only more laborious, and we prefer to skip it. This proves that $V(2) \cap I_{t}(K)$ contains no other elements than those generated by $g_{1}$ and $g_{2}$. 
(3) In Propositions 5.6 and 5.10 we have found elements in the peripheral ideal that are of the form $(3,0)_{T}+v_{0}$ and $(3,1)_{T}+v_{1}$, with $v_{0}, v_{1} \in V(2)$. The productto-sum formula

$$
(3, q+1)_{T}=t^{3}(0,1)_{T} *(3, q)_{T}-t^{6}(3, q-1)_{T}
$$

allows us to construct, for any integer $q$, an element in the peripheral ideal of the form $(3, q)_{T}+v_{3, q}$ with $v_{3, q} \in V(2)$. Also, the product-to-sum formula

$$
(p+1, q)_{T}=t^{3 q}(p-2, q)_{T} *(3,0)_{T}-t^{6 q}(p-5, q)_{T}
$$

allows us to construct, for any integers $p \geq 4$ and $q$, an element in the peripheral ideal of the form $(p, q)_{T}+v_{p, q}$, with $v_{p, q} \in V(p-1)$. If there existed an element in the peripheral ideal that is not generated by the four exhibited in the statement, then we could reduce it using elements of the form $(p, q)_{T}+v_{p, q}$ to one in $V(2)$. But all elements in $V(2) \cap I_{t}(K)$ are generated by $g_{1}$ and $g_{2}$. Hence the conclusion.

\section{The noncommutative A-IDEAL OF The Figure-EIGHT KNOt}

The noncommutative A-ideal is obtained from the peripheral ideal by extending it to the ring of trigonometric polynomials in the noncommutative torus, and then contracting it to the quantum plane. Specifically, in the four generators exhibited in Propositions 5.4 5.6 and 5.10 we replace each $(p, q)_{T}$ by $t^{-p q}\left(l^{p} m^{q}+l^{-p} m^{-q}\right)$ to obtain a Laurent polynomial in the noncommuting variables $l$ and $m$ with coefficients in $\mathbb{C}(t)$. Then, we multiply these Laurent polynomials on the left by appropriate powers of $l$ and $m$ to obtain polynomials in $\mathbb{C}_{t}[l, m]$. These four polynomials are the generators of the noncommutative A-ideal.

In Lemma 5.5 we saw that $x Y$ and $x Z$ are the images of two elements from the Kauffman bracket skein module of the torus. We identified $x Y$ and $x Z$ with their preimages. Now extend those to the noncommutative torus, to obtain

$$
\begin{aligned}
x & =\frac{1}{\left(t^{16}-1\right)\left(1-t^{-4}+t^{-8}\right)}\left[\left(-1+t^{-4}\right)\left(l^{2} m+l^{-2} m^{-1}\right)+t^{8}\left(l^{2} m^{-1}+l^{-2} m\right)\right. \\
& +\left(-t^{4}+1\right)\left(l m^{5}+l^{-1} m^{-5}\right)+\left(t^{8}+t^{6}-t^{2}\right)\left(l m^{3}+l^{-1} m^{-3}\right) \\
& +\left(-t^{10}-t^{8}+t^{6}-t^{-4}+t^{-8}\right)\left(l m+l^{-1} m^{-1}\right) \\
& +\left(t^{-2}-t^{16}+t^{12}-t^{8}-t^{4}+1-t^{-4}\right)\left(l m^{-1}-l^{-1} m\right) \\
& +\left(-t^{2}-1+t^{-4}\right)\left(l m^{-3}+l^{-1} m^{3}\right)+t^{4}\left(l m^{-5}+l^{-1} m^{5}\right) \\
& +\left(-t^{1} 2+t^{8}+t^{-4}\right)\left(m^{5}+m^{-5}\right)+\left(2 t^{12}-2 t^{8}+t^{4}-2 t^{-4}+t^{-8}+1\right)\left(m^{3}+m^{-3}\right) \\
& \left.+\left(-t^{16}+t^{8}+t^{4}+2+2 t^{-4}+t^{-8}\right)\left(m+m^{-1}\right)\right] .
\end{aligned}
$$

and

$$
\begin{aligned}
& x Z=\frac{1}{\left(t^{-16}-1\right)\left(1-t^{4}+t^{8}\right)}\left[\left(t^{4}-1\right)\left(l^{2} m^{-1}+l^{-2} m\right)+t^{-8}\left(l^{2} m+l^{-2} m^{-1}\right)\right. \\
& \quad+\left(-t^{-4}+1\right)\left(l m^{-5}+l^{-1} m^{5}\right)+\left(t^{-8}+t^{-6}-t^{-2}\right)\left(l m^{-3}+l^{-1} m^{3}\right) \\
& \quad+\left(-t^{-10}-t^{-8}+t^{-6}-t^{-4}-t^{4}+t^{8}\right)\left(l m^{-1}+l^{-1} m\right) \\
& \quad+\left(t^{2}-t^{-16}+t^{-12}-t^{-8}-t^{-4}+1-t^{4}\right)\left(l m+l^{-1} m^{-1}\right) \\
& \quad+\left(-t^{-2}-1+t^{4}\right)\left(l m^{3}+l^{-1} m^{-3}\right)+t^{-4}\left(l m^{5}+l^{-1} m^{-5}\right) \\
& +\left(t^{-12}+t^{-8}+t^{4}\right)\left(m^{5}+m^{-5}\right)+\left(2 t^{-12}-2 t^{-8}+t^{-4}-2 t^{4}+t^{8}+1\right)\left(m^{3}+m^{-3}\right) \\
& \left.\quad+\left(-t^{-16}+t^{-8}+t^{-4}+2+2 t^{4}+t^{8}\right)\left(m+m^{-1}\right)\right] .
\end{aligned}
$$


Then in view of Lemma 5.8 we can write

$$
\begin{aligned}
Y & =\frac{1}{2\left(t^{-8}-t^{4}\right)\left(t^{4}-1\right)}\left[\left(t^{4}+t^{-4}+1-2 t^{8}-t^{-8}\right)\left(m+m^{-1}\right)(x Y)\right. \\
& +\left(t^{-4}+1-t^{4}-t^{8}\right)\left(m+m^{-1}\right)(x Z)-\left(l^{2}+l^{-2}\right) \\
& -t^{2}\left(l m^{4}+l^{-1} m^{-4}\right)-t^{-2}\left(l m^{-4}+l^{-1} m^{4}\right) \\
& +t^{2}\left(l m^{2}+l^{-1} m^{-2}\right)+t^{-2}\left(l m^{-2}+l^{-1} m^{2}\right) \\
& +\left(t^{6}-t^{-6}\right)\left(l+l^{-1}\right)-\left(t^{8}+t^{-8}\right)\left(m^{4}+m^{-4}\right) \\
& \left.+\left(t^{-4}-t^{4}+2-t^{8}+t^{-8}\right)\left(m^{2}+m^{-2}\right)+\left(2 t^{-4}+2-2 t^{8}\right)\right]
\end{aligned}
$$

and

$$
\begin{aligned}
Z & =\frac{1}{2\left(t^{8}-t^{-4}\right)\left(t^{-4}-1\right)}\left[\left(t^{-4}+t^{4}+1-2 t^{-8}-t^{8}\right)\left(m+m^{-1}\right)(x Z)\right. \\
& +\left(t^{4}+1-t^{-4}-t^{-8}\right)\left(m+m^{-1}\right)(x Y)-\left(l^{2}+l^{-2}\right) \\
& -t^{2}\left(l m^{4}+l^{-1} m^{-4}\right)-t^{-2}\left(l m^{-4}+l^{-1} m^{4}\right) \\
& +t^{2}\left(l m^{2}+l^{-1} m^{-2}\right)+t^{-2}\left(l m^{-2}+l^{-1} m^{2}\right) \\
& +\left(t^{-6}-t^{6}\right)\left(l+l^{-1}\right)-\left(t^{8}+t^{-8}\right)\left(m^{4}+m^{-4}\right) \\
& \left.+\left(t^{4}-t^{-4}+2-t^{-8}+t^{8}\right)\left(m^{2}+m^{-2}\right)+\left(2 t^{4}+2-2 t^{-8}\right)\right]
\end{aligned}
$$

From these formulas and Theorem 5.11 we obtain the following

Theorem 6.1. The noncommutative A-ideal of the figure-eight knot is generated by the following four elements

$$
\begin{aligned}
t^{-18} & l^{3} m^{14}+t^{8} l^{2} m^{14}-t^{-18} l^{3} m^{12}+\left(t^{4}+t^{-4}-2 t^{8}\right) l^{2} m^{12}-t^{18} l m^{12}+t^{-40} l^{4} m^{10} \\
& +\left(-t^{-22}-t^{-18}+t^{-14}-t^{-6}\right) l^{3} m^{10}+\left(t^{8}-t^{4}+t^{-4}-1-t^{12}\right) l^{2} m^{10} \\
& +\left(t^{30}+t^{18}\right) l m^{10}+\left(-t^{-22}+t^{-6}-t^{-10}\right) l^{3} m^{8}+\left(-t^{8}+1+t^{-4}+t^{12}\right) l^{2} m^{8} \\
& +\left(t^{6}+2 t^{22}-t^{14}-t^{6}+t^{18}\right) l m^{8}+t^{-20} l^{4} m^{6} \\
& +\left(-t^{-2}+t^{-22}-t^{-14}+2 t^{-6}+t^{-10}+t^{-4}\right) l^{3} m^{6}+\left(1+t^{12}-t^{8}+t^{-4}\right) l^{2} m^{6} \\
& +\left(t^{22}-t^{6}-t^{18}+t^{-4}\right) l m^{6}+\left(t^{2}+t^{-10}\right) l^{3} m^{4}+\left(t^{-2}+t^{8}-t^{4}+t^{-4}-t^{12}\right) l^{2} m^{4} \\
& +\left(-t^{6}-t^{10}+t^{14}-t^{22}\right) l m^{4}+t^{16} m^{4}-t^{-10} l^{3} m^{2}+\left(t^{4}-t^{-4}-2 t^{8}\right) l^{2} m^{2}-t^{10} l m^{2} \\
& +t^{8} l^{2}+t^{10} l, \\
& \\
& \\
t^{-8} & l^{2} m^{14}+t^{18} l m^{14}-t^{-18} l^{3} m^{12}+\left(t^{4}+t^{-4}-2 t^{-8}\right) l^{2} m^{12}-t^{18} l m^{12} \\
& +\left(t^{-30}+t^{-18}\right) l^{3} m^{10}+\left(t^{4}+t^{-8}-1-t^{-4}-t^{-12}\right) l^{2} m^{10} \\
& +\left(t^{14}-t^{-22}-t^{6}-t^{18}\right) l m^{10}+t^{40} m^{10}-t^{-36} l^{4} m^{8} \\
& +\left(-t^{-14}-t^{-26}+t^{6}+t^{-18}+2 t^{-22}\right) l^{3} m^{8}+\left(t^{-12}+t^{4}+1-t^{-8}\right) l^{2} m^{8} \\
& +\left(-t^{22}-t^{10}+t^{6}\right) l m^{8}+\left(t^{-22}-t^{-18}-t^{-6}\right) l^{3} m^{6}+\left(1+t^{4}+t^{-12}-t^{-8}\right) l^{2} m^{6} \\
& +\left(-t^{24}+2 t^{6}-t^{2}+t^{10}\right) l m^{6}-t^{-20} m^{6}+t^{-16} l^{4} m^{4} \\
& +\left(-t^{-22}-t^{-6}-t^{-10}+t^{-14}\right) l^{3} m^{4}+\left(t^{4}-t^{-12}+t^{-8}-t^{-4}-1\right) l^{2} m^{4} \\
& +\left(t^{-2}+t^{10}\right) l m^{4}-t^{-10} l^{3} m^{2}+\left(t^{-4}-2 t^{-8}-t^{4}\right) l^{2} m^{2}-t^{10} l m^{2}+t^{-8} l^{2}+t^{-10} l^{3}
\end{aligned}
$$




$$
\begin{aligned}
& l^{3} m^{7}\left[-t^{-2}\left(l^{3} m+l^{-3} m^{-1}\right)+\left(t^{16}-1\right)\left(t^{4}+1\right) t^{-2}\left(m^{2}+m^{-2}+t^{-4}-1\right)(x Y)\right. \\
& +\left(t^{16}-1\right) t^{-2}\left(m^{2}+m^{-2}+t^{-4}-t^{-8}\right)(x Z)-t^{2}\left(l^{2} m^{5}+l^{2} m^{-5}\right) \\
& +t^{2}\left(l^{2} m^{3}+l^{-2} m^{-3}\right)+\left(t^{-2}+t^{2}-t^{8}\right)\left(l^{2} m+l^{-2} m^{-1}\right) \\
& +\left(t^{-2}-t^{14}\left(l^{2} m^{-1}+l^{-1} m^{2}\right)-t^{-2}\left(l^{2} m^{-3}+l^{-2} m^{3}\right)\right. \\
& -t^{10}\left(l m^{5}+l^{-1} m^{-5}\right)+\left(t^{-6}-t^{6}-t^{14}\right)\left(l m^{3}+l^{-1} m^{-3}\right) \\
& +\left(t^{-6}-t^{6}-2 t^{11}-2 t^{15}+t^{-1}\right)\left(l m+l^{-1} m^{-1}\right) \\
& +\left(t^{-6}-t^{-2}-1-t^{6}-t^{14}\right)\left(l m^{-1}-l^{-1} m\right)+\left(t^{-6}-t^{-2}-t^{6}\right)\left(l m^{-3}-l^{-1} m^{3}\right) \\
& -t^{10}\left(l m^{-5}+l^{-1} m^{5}\right)-\left(t^{2}-t^{-2}\left(m^{3}-m^{-3}\right)\right. \\
& \left.+\left(t^{18}+t^{14}+2 t^{10}-t^{8}+t^{6}-2 t^{-6}-t^{-10}\right)\left(m+m^{-1}\right)\right],
\end{aligned}
$$

and

$$
\begin{aligned}
& l^{3} m^{10}\left[-t^{3}\left(l^{3}+l^{-3}\right)+\left(-t^{-11}\left(l m^{4}+l^{-1} m^{-4}\right)-t^{-11}\left(l m^{-2}+l^{-1} m^{2}\right)\right.\right. \\
& \left.-\left(t^{5}+t^{-11}+t^{-7}\right)\left(l m^{2}+l^{-1} m^{-2}\right)-\left(t^{5}+t^{-11}+t^{-7}\right)\left(l+l^{-1}\right)\right) Y \\
& +\left(-t^{-11}\left(l m^{4}+l^{-1} m^{-4}\right)-t^{-11}\left(l m^{-2}+l^{-1} m^{2}\right)\right. \\
& \left.-\left(t^{5}+t^{-11}+t\right)\left(l m^{2}+l^{-1} m^{-2}\right)-\left(t^{5}+t^{-11}+t\right)\left(l+l^{-1}\right)\right) Z \\
& -t^{-1}\left(l^{2} m^{4}+l^{-2} m^{-4}\right)-t^{-5}\left(l m^{-4}+l^{-1} m^{4}\right)+t^{-1}\left(l^{2} m^{2}+l^{-2} m^{-2}\right) \\
& +\left(t^{-1}+t^{-5}\right)\left(l^{2}+l^{-2}\right)-\left(t^{5}-t^{-7}\right)\left(l m^{4}+l^{-1} m^{-4}\right) \\
& -\left(t^{5}+t^{-1}+t^{-7}\left(l m^{-2}+l^{-1} m^{2}\right)-t^{-11}\left(l m^{6}+l^{-1} m^{-6}\right)\right. \\
& -t^{-11}\left(l m^{-4}+l^{-1} m^{4}\right)+\left(t^{-11}-t^{-1}\right)\left(l m^{2}+l^{-1} m^{-2}\right) \\
& \left.+\left(t^{-11}-t\right)\left(l+l^{-1}\right)-t^{-1}\left(m^{6}+m^{-6}\right)+t^{-1}\left(m^{4}+m^{-4}\right)+t^{-1}\left(m^{2}+m^{-2}\right)+2 t^{3}\right] .
\end{aligned}
$$

\section{The A-IDeal Determines the Jones polynomial}

For a knot $K$ we denote by $\kappa_{n}(K)$ the $n$th colored Kauffman bracket, that is, the Kauffman bracket of the skein in the 3 -dimensional sphere which is $K$ colored by the $n$th Jones-Wenzl idempotent. As such, $\kappa_{1}(K)$ is the classical Kauffman bracket of the knot. There is a framing ambiguity in this definition, which we eliminate by always working with the zero framing of the knot.

Theorem 7.1. If a knot has the same noncommutative A-ideal as the figure-eight knot, then all colored Kauffman brackets are the same.

Proof. Two knots that have the same noncommutative A-ideals have the same peripheral ideals (see [7]). It has been shown in [9] that every element of the form $\sum_{i} c_{i}\left(p_{i}, q_{i}\right)_{T}$ in the peripheral ideal gives rise to a family of relations of the form

$$
\begin{aligned}
& \sum_{i} c_{i}\left(t^{\left(2 n+p_{i}\right) q_{i}}\left[\left(-t^{2}\right)^{q_{i}} \kappa_{p_{i}+n}(K)-\left(-t^{-2}\right)^{q_{i}} \kappa_{p_{i}+n-2}(K)\right]\right. \\
& \left.+t^{-\left(2 n-p_{i}\right) q_{i}}\left[\left(-t^{2}\right)^{q_{i}} \kappa_{p_{i}-n}(K)-\left(-t^{-2}\right)^{q_{i}} \kappa_{p_{i}-n-2}(K)\right]\right)=0 .
\end{aligned}
$$

This is the "orthogonality relation between the A-ideal and the Jones polynomial" discovered in [7]. These relations indexed by the nonnegative integer $n$ allow us to compute $\kappa_{n}(K)$ recursively. Of course, one should note that $\kappa_{0}(K)=1, \kappa_{-1}(K)=$ $0, \kappa_{-n-2}(K)=-\kappa_{n}(K)$ for all $n$ and all knots.

Let $K$ be the figure eight knot and consider the element provided by Proposition [5.6. multiplied by $-t^{-1}$ so that the coefficient of $(3,1)_{T}$ is 1 . The coefficient of $\kappa_{3}(K)$ in the recursive relation with $n=0$ is $-2 t^{5}$, which is nonzero, so $\kappa_{3}(K)$ can be 
determined in terms of $\kappa_{2}(K), \kappa_{1}(K), \kappa_{0}(K)=1, \kappa_{-1}(K)=0$ and $\kappa_{-2}(K)=-1$. For $n>1$, the coefficient of $\kappa_{n+3}(K)$ is $-t^{2 n+5}$, which is always nonzero, so we can compute $\kappa_{n+3}(K), n \geq 0$, in terms of colored Kauffman brackets of lower color.

On the other hand, an element of the form

$$
\sum_{q} c_{2, q}(2, q)_{T}+\sum_{q} c_{1, q}(1, q)_{T}+\sum_{q} c_{0, q}(0, q)_{T}
$$

in the peripheral ideal gives rise to an orthogonality relation of the form

$$
\left[\sum_{q}(-1)^{q} c_{2, q}\left(t^{4 q}+1\right)\right] \kappa_{2}(K)+\left[\sum_{q}(-1)^{q} c_{1, q}\left(t^{3 q}+t^{q}\right)\right] \kappa_{1}(K)+p(t)=0,
$$

where $p(t)$ is a Laurent polynomial in $t$.

Hence the elements provided by Proposition 5.4 give rise to orthogonality relations of the form

$$
\begin{aligned}
& P_{1}(t) \kappa_{2}(K)+Q_{1}(t) \kappa_{1}(K)=R_{1}(t) \\
& P_{2}(t) \kappa_{2}(K)+Q_{2}(t) \kappa_{1}(K)=R_{2}(t) .
\end{aligned}
$$

with $P_{i}(t), Q_{i}(t), R_{i}(t), i=1,2$, Laurent polynomials in $t$. View this as a linear system of equations in the unknowns $\kappa_{2}(K)$ and $\kappa_{1}(K)$.

One easily computes $P_{1}(t)=-t^{-6}+t^{2}$ and $P_{2}(t)=-t^{6}+t^{-2}$, so $P_{2}(t)=$ $\left(-t^{4}\right) P_{1}(t)$. Hence the system has zero determinant only if $Q_{2}(t)=\left(-t^{4}\right) Q_{1}(t)$. But the term of highest degree in $Q_{1}(t)$ is $-t^{24}$ while the term of highest degree in $Q_{2}(t)$ is $t^{16}$, so equality is impossible.

It follows that $\kappa_{1}(K)$ and $\kappa_{2}(K)$ are uniquely determined by the two orthogonality relations, and the conclusion of the theorem follows.

Corollary 7.2. Any knot having the same noncommutative A-ideal as the figureeight knot has the same Jones polynomial.

In fact F. Nagasato wrote down the recursive relation obtained from the first element of Proposition 5.4 and checked that the recursion yields Habiro's formulas for the colored Kauffman brackets of the figure-eight knot.

We have seen that the property stated in the above theorem is shared by the unknot 9 and $(2,2 p+1)$-torus knots 10 . There the property followed from the existence of a polynomial of second degree in $l$ in the noncommutative A-ideal. The present result shows that this condition is not necessary. In 9] it was also proved that for any knot, the noncommutative A-ideal together with finitely many colored Kauffman brackets determine all other colored Kauffman brackets of the knot. All these facts suggest the following

Conjecture. The noncommutative A-ideal of a knot determines all its colored Kauffman brackets. In particular if two knots have the same noncommutative Aideal then they have the same Jones polynomial.

\section{REFERENCES}

[1] D. Bullock, The $(2, \infty)$-skein module of the complement of a $(2,2 p+1)$ torus knot, J. Knot Theory Ramifications 4(1995), no. 4, 619-632.

[2] D. Bullock, Rings of $\mathrm{Sl}_{2}(\mathbb{C})$-characters and the Kauffman bracket skein module, Comment. Math. Helv. 72 (1997), no. 4, 521-542 q-alg/9604014

[3] D. Bullock, W. Lofaro, The Kauffman bracket skein module of a twist knot exterior, preprint. 
[4] M. Culler, P. Shalen, Varieties of group representations and splittings of 3-manifolds, Ann. of Math., 117 (1983), 109-146.

[5] D. Cooper, M. Culler, H. Gillett, D.D. Long, P.B. Shalen, Plane Curves associated to character varieties of 3-manifolds, Inventiones Math. 118, pp. 47-84 (1994).

[6] Ch. Frohman, R. Gelca, Skein modules and the noncommutative torus, Transactions Amer. Math. Soc., 352(2000), 4877-4888 math.QA/9806107

[7] Ch. Frohman, R. Gelca, W. Lofaro, The A-polynomial from the noncommutative viewpoint, Transactions Amer. Math. Soc., 354(2001), 735-747, math.QA/9812048

[8] R. Gelca, Noncommutative trigonometry and the A-polynomial of the trefoil knot, Math. Proc. Cambridge Phil. Soc., 133(2002), 311-323, math.GT/0004155

[9] R. Gelca, On the relation between the A-polynomial and the Jones polynomial, Proceedings Amer. Math. Soc., 130(2001), 1235-1241 math.GT/0004158

[10] R. Gelca, J. Sain, The noncommutative A-ideal of a $(2,2 p+1)$-torus knot determines its Jones polynomial, J. Knot Theory and Ramifications, to appear math.GT/0201100

[11] L. Kauffman, State models and the Jones polynomial, Topology 26 no. 3 (1987) 395-401.

[12] F. Nagasato, An approach to the A-polynomial of $(2,2 p+1)$-torus knots from the FrohmanGelca-Lofaro theory, preprint.

[13] J. H. Przytycki, Skein modules of 3-manifolds, Bull. Pol. Acad. Sci. 39(1-2) (1991) 91-100.

[14] J.H. Przytycki, A.S. Sikora, On Skein Algebras And $S_{2}(\mathbb{C})$-Character Varieties, Topology 39(2000), 115-148 q-alg/9705011

[15] V.G. Turaev, Algebras of loops on surfaces, algebras of knots, and quantization, Adv. Ser. in Math. Physics 9 (1989), ed. C. N. Yang, M. L. Ge, 59-95.

Department of Mathematics and Statistics, Texas Tech University, Lubbock, TX 79409 and Institute of Mathematics of the Romanian Academy, Bucharest, Romania

E-mail address: rgelca@math.ttu.edu

Department of Mathematics, University of California at Berkeley, Berkeley, CA 94720

E-mail address: jsain@Math.Berkeley.EDU 